\title{
CHARACTERISATION OF HORDEUM VULGARE CELLULOSE SYNTHASE-LIKE F6 PROMOTER VIA TRANSGENE EXPRESSION IN RICE
}

\author{
Azreena Jamahari ${ }^{1 a}$, Wong Ling Chie ${ }^{2 a}$, Fan Xiaolai ${ }^{3 b}$, Liu Qiaoquan ${ }^{4 b}$, Leong Sui Sien ${ }^{5 c}$, \\ Amy Halimah Rajaee ${ }^{6 c}$, Fauziah Abu Bakar ${ }^{7 d}$, Patricia King Jie Hung ${ }^{8 d}$, Hairul Azman \\ Roslan $^{9 e}$ and Wong Sie Chuong ${ }^{10 a^{*}}$
}

\begin{abstract}
aDepartment of Science and Technology, Faculty of Humanities, Management and Science, Universiti Putra Malaysia Bintulu Sarawak Campus, 97000 Sarawak, MALAYSIA. E-mail: azreena.jamahari@gmail.com; vikki.w007@gmail.com²; wongsie@upm.edu.my ${ }^{10}$

${ }^{b}$ Key Laboratory of Plant Functional Genomics of the Ministry of Education, College of Agriculture, Yangzhou University, Yangzhou, 225009, CHINA. Email: xlfan@yzu.edu.cn ${ }^{3}$; qqliu@yz.edu.cn ${ }^{4}$

cDepartment of Animal Science and Fisheries, Faculty of Agricultural Science and Forestry, Universiti Putra Malaysia Bintulu Sarawak Campus, 97000 Sarawak, MALAYSIA. Email: leongsuisien@upm.edu.my ${ }^{5}$; amyhalimah@upm.edu.my ${ }^{6}$

${ }^{\mathrm{d} D e p a r t m e n t}$ of Crop Science, Faculty of Agricultural Science and Forestry, Universiti Putra Malaysia Bintulu Sarawak Campus, 97000 Sarawak, MALAYSIA. Email: ab_fauziah@upm.edu.my ${ }^{7}$; patricia@upm.edu.my ${ }^{8}$ eFaculty of Resource Science and Technology, Universiti Malaysia Sarawak, 94300 Kota Samarahan, MALAYSIA. Email: rhairul@unimas.my ${ }^{9}$

*Corresponding Author: wongsie@upm.edu.my

Received: $10^{\text {th }}$ Feb $2021 \quad$ Accepted: $16^{\text {th }}$ Jun 2021

Published: $30^{\text {th }}$ Jun 2021
\end{abstract}

DOI: https://doi.org/10.22452/mjs.vol40no2.6

\begin{abstract}
Beta-glucan in cereal crops is known as a functional food, which can reduce cardiovascular diseases by lowering blood cholesterol levels. However, beta-glucan content is relatively low in rice grains, despite being relatively abundant in barley and oat grains. Taking advantage of rice as the staple food for Asians, increasing beta-glucan content in rice for their consumption may help to reduce cardiovascular-related diseases among them. Previous attempts in increasing beta-glucan content in rice via transgene expression of betaglucan synthase genes from barley into rice were unsuccessful due to the use of non-tissue specific as well as constitutively expressing promoter. The current transgenic expression study was performed to characterise the promoter of beta-glucan synthase gene in barley using betaglucuronidase (GUS) reporter gene. Two fragments of $H v C s l F 6$ promoter (2771 bp and $1257 \mathrm{bp}$ ) were successfully fused with GUS reporter gene and integrated into rice plants, demonstrated that the promoter was functional in the heterologous plant system. The presence of blue GUS staining was observed on the leaf, root, stem, and grain of the transgenic rice regardless of the promoter length used and stayed functional up to the next generation. GUS qualitative analysis confirmed that the shorter promoter length generated a stronger GUS activity in comparison to the longer one. This indicated that the presence of repressor elements in between the -2771 bp and -1257 bp regions. The preliminary results shed light on the strong promoter activity in the rice endosperm tissue. It can become an alternative to the collection of plant promoters that can be used for grain quality improvement and biofortification.
\end{abstract}

Keywords: $H v C s l F 6$ promoter, endosperm-specific, transgenic rice 


\section{INTRODUCTION}

Beta-glucan is a hemicellulosic polysaccharide found in the cell wall of grass and cereal crops. It is made up of $\beta$-D glucose monomers bounded by $\beta$-1.3- and $\beta$-1.4- glycosidic linkages. The random insertion of 1,3-glycosidic bond between the 1,4-glycosidic linkage prevents the proper superimposition of the linear polymer. The relaxed beta-glucan structure leads to an increase in water solubility and viscosity when being hydrated. Beta-glucan derived from different cereal plants possesses different water-solubility properties. Barley and oat have abundant beta-glucan in their grains which is beneficial in improving human overall health and wellbeing (Marković et al., 2017).

Highly soluble beta-glucan is claimed to have prebiotic properties. This is due to the ability of the beta-glucan to resist gastrointestinal enzymes and promote the growth of probiotic bacteria such as Bifidobacterium animalis, Lactobacilles casei, Lactobacillus bulgaricus, and Bifidobacterium adolescentis (Arena et al., 2014; Ren et al., 2018). The improvement of the gut microbiota composition promotes systemic immunity effects. Aside from that, oat and barley beta-glucan can reduce blood cholesterol levels. The oat beta-glucan increases the viscosity of gut contents and modulates the bile acid metabolism (Joyce et al., 2019). The bile acid is excreted out from the body through fecal to prevent reabsorption in the terminal ileum (Wolever et al., 2010; Joyce et al., 2019). Consequently. the conversion rate of cholesterol to bile acid is increased while cholesterol level in the blood vessel is reduced (Joyce et al., 2019).

The cellulose synthase-like F6 (CslF6) gene has been discovered to direct the beta-glucan in several cereal plants including Brachypodium distachyon and Setaria viridis (Kim et al., 2018; Ermawar et al., 2015). The CslF6 gene knockout studies in rice, wheat, and barley demonstrated a significant reduction of beta-glucan in the host plants. On the other hand, overexpression of the CslF6 gene in the host plant increased beta-glucan content in both homologous and heterologous plant systems (Burton et al., 2011; Vega-Sánchez et al., 2012; Kim et al., 2018; Lim et al., 2019). These results confirmed the essential role of the CslF6 gene in the production of beta-glucan. Moreover, there are other betaglucan synthase identified from the cellulose synthase-like family genes. Expression of $\mathrm{HvCslHI}$ gene into Nicotiana benthamiana produced beta-glucan in the cell wall of the transgenic leaf (Wilson et al., 2015; Little et al., 2018). Burton et al. (2011) demonstrated that $H v C s l F 3$ and $\mathrm{HvCslF9}$ genes also increased beta-glucan content in the grain of transgenic barley. To investigate the individual role of the genes in beta-glucan production, the genes need to be expressed in beta-glucan devoid of host plants.

Insertion of $\mathrm{HvCslF6}$ gene into transgenic barley under the direction of CaMV $35 s$ promoter caused the plant to die due to a high amount of viscous beta-glucan in the vascular plant tissues (Burton et al., 2011). It caused limited transportation of the nutrient and water to other parts of the plants, which rendered a normal metabolism regulation (Burton et al., 2011). The problem was solved using endospermspecific Asglol promoter, which reduced the plant mortality although an adverse phenotype effect was observed in the transgenic grains. Beta-glucan accumulation in the endosperm tissue reduced the amount of starch available, affecting the appearance and shape of the grain (Burton et al., 2011). Thus, the usage of endosperm-specific promoter with temporal specific characteristics is ideal to 
introduce high transgene expression in the host grain without disturbing the grain development and maturity.

A previous study indicated that the $H v C s l F 6$ promoter was unable to drive the expression of Luciferase reporter gene in the transgenic Nicotiana benthamiana. However, it successfully elicited the reporter gene expression in transiently transformed barley coleoptile, root, and first base leaf (Dimitroff, 2016). Due to the inconsistent findings between these two host plants, the expression pattern of the $\mathrm{HvCslF6}$ promoter can be further studied by expressing the reporter gene into other cereal plants that lack beta-glucan such as rice. Thus, this study investigated the functional region of the $H v C s F 6$ promoter and its expression pattern in the rice host plant.

\section{MATERIALS AND METHODS}

\subsection{Plant materials}

Barley (Hordeum vulgare cv. Sloop) leaves were used for promoter isolation. Meanwhile, rice (Oryza sativa $L$. ssp. Japonica cv. Nipponbare) plants were used for rice transformation mediated by Agrobacterium tumefaciens.

\subsection{Isolation and bioinformatic analysis of $\mathrm{HvCslF} 6$ promoter}

Genomic DNA of barley was extracted from 2 weeks old barley leaves using a Plant DNA extraction kit from Vivantis (Selangor, Malaysia). The promoter was isolated using PCR procedure according to the manufacturer's guidelines. The primers used were designed using Geneious 9.0 software from Biomatters Ltd. (Auckland, New Zealand) based on the 2771 bp upstream of transcriptional start site (TSS) of CslF6 gene of barley Morex cultivar DNA sequence as assembled in contig_41513 in Morex Genes-Barley RNA-seq database (https://ics.hutton.ac.uk/morexGenes). The PCR was conducted through initial denaturation at $98{ }^{\circ} \mathrm{C}$ for $30 \mathrm{sec}$, followed by 30 cycles of $98^{\circ} \mathrm{C}$ for $10 \mathrm{sec}, 60^{\circ} \mathrm{C}$ for $30 \mathrm{sec}$, and $72{ }^{\circ} \mathrm{C}$ for $90 \mathrm{sec}$. The final extension was conducted at $72^{\circ} \mathrm{C}$ for $5 \mathrm{~min}$ using Phusion High-Fidelity DNA polymerase from NEB (Ipswich, MA). The primers used were F6PromFor (5'AGGAAAAACCCTGTGCCACA -3') and F6PromRev CATGGCCGTCGTCCTCAAT-3'). The 2771 bp PCR product was gel electrophoresed on 1\% agarose gel, purified using QiaQuick Gel extraction kit from Qiagen (Hilden, Germany), and sent for DNA sequencing service (Apical Scientific Sdn Bhd, Malaysia). The DNA sequence was analysed and aligned with the HvCslF6 promoter sequence of other barley cultivars available in Wong et al. (2015) using Geneious 9.0 software. The DNA sequence was then analysed in-silico using PLACE (Higo et al., 1998) and PlantCARE (Lescot et al., 2002) databases to identify the endosperm-specific regulatory elements.

\subsection{Plant expression vector construction}

Two different lengths (2771 bp and $1257 \mathrm{bp)}$ of HvCslF6 promoter were analysed in this study. Primers P1 and P2 were used to amplify the first fragment whereas primers P3 and P4 were used to amplify the second fragment 2771bp HvCslF6 promoter (Figure 1). Meanwhile, primers P4 and P5 were used to amplify the $1257 \mathrm{bp} \mathrm{HvCslF6}$ promoter. All primers were designed using NEBuilder Assembly tool version 2.2.8 (Ipswich, MA) to recombine the pCAMBIA1305.1 digested plasmid with the interested insert fragment. The DNA sequence of the primers used to amplify the fragments was demonstrated in 
Table 1. The inserted fragments were amplified using Phusion High-Fidelity DNA polymerase kit, following the PCR condition of initial denaturation of $98^{\circ} \mathrm{C}$ for $30 \mathrm{sec}, 30$ cycles of $98^{\circ} \mathrm{C}$ for $10 \mathrm{sec}, 68^{\circ} \mathrm{C}$ for $30 \mathrm{sec}$, and $72{ }^{\circ} \mathrm{C}$ for $90 \mathrm{sec}$. The correct sized fragments were checked on $1 \% \mathrm{w} / \mathrm{v}$ agarose gel and purified using QiaQuick Gel extraction kit.

Table 1. List of primer used to amplify $2771 \mathrm{bp}$ and $1257 \mathrm{bp} H v C s l F 6$ promoter.

\begin{tabular}{ccc} 
Primer & Orientation & DNA sequence (5' to 3') \\
\hline P1 & Forward & CAGCTATGACCATGATTACGAGGAAAAACCCTGTG \\
P2 & Reverse & AGGAATGCATTGGTCCCCTG \\
P3 & Forward & GGGACCAATGCATTCCTTCTCGTG \\
P4 & Reverse & TAGAAATTTACCCTCAGATCTACCATGGCCGTCGTCGT \\
P5 & Forward & CAGCTCA
\end{tabular}

Bold sequences are homologous to the linearised vector

The pCAMBIA1305.1 plant vector was linearised using ECoRI and BglII restriction enzymes from Promega (Madison, WI) and then purified using a QiaQuick Gel extraction kit. The HvCslF6 promoter was fused with the GUS reporter gene by Hot Fusion cloning method with slight modification (Fu et al., 2014). About 250-300 ng of linearised vector and 20-30 ng of inserts were added into a PCR microcentrifuge tube containing $5 \mu \mathrm{L}$ of $2 \mathrm{X}$ Hot Fusion buffer. The sterile ultrapure water was added to make a total reaction volume of $10 \mu \mathrm{L}$. For multiple fragments assembly, $50 \mathrm{ng}$ of each insert was used while the amount of digested vector used remained the same. The tubes were incubated at $50{ }^{\circ} \mathrm{C}$ for $60 \mathrm{~min}$. An immediate use of $5 \mu \mathrm{L}$ of the Hot Fusion reaction for bacterial heat-shock transformation according to Sambrook et al. (1989). The survived bacteria colony was further confirmed using colony PCR, amplifying ends of pCAMBIA1305.1 vector covering the $H v C s l F 6$ promoter region. The amplification was performed by using Gotaq green master PCR kit from
Promega (Madison, WI) with primers Cpcf (5'-AAACCGCCTCCCCGCGCGTT-3') and Cpcr (5'GGTACAGACTAGTTCGTC-3'). Both primers were flagging on the end flap of digested pCAMBIA1305.1 to confirm the correct size of the inserted DNA fragment after DNA recombination and bacteria cloning steps. The PCR was conducted with the following PCR amplification conditions: $95{ }^{\circ} \mathrm{C}$ for $3 \mathrm{~min}, 30$ cycles of $9{ }^{\circ} \mathrm{C}$ for $45 \mathrm{sec}, 57^{\circ} \mathrm{C}$ for $45 \mathrm{sec}$, and $72{ }^{\circ} \mathrm{C}$ for $2 \mathrm{~min}$. The final extension was $72^{\circ} \mathrm{C}$ for $10 \mathrm{~min}$. Transformed bacteria with correct insert size were grown on Luria-Bertani (LB) broth (Merck, Germany) at $37{ }^{\circ} \mathrm{C}$ overnight and the plasmids were extracted using High purity plasmid miniprep kit from Dongsheng Biotech (Guangzhou, China) before sent for sequencing to confirm the plasmid assembly.

\subsection{Agrobacterium-mediated rice transformation}

The 2771 bp and 1257 bp HvCslF6prom fused with GUS reporter 
gene expression vectors were transformed into Agrobacterium tumefaciens strain EHA105 via electroporation according to the standard protocol of MicroPulser electroporation system from Bio-Rad Laboratories (Hercules, CA). Five survived bacterial colonies were subjected to colony PCR procedure using a Gotaq DNA polymerase kit, according to the manufacturer's protocol with modification. The PCR was conducted with the following PCR amplification conditions: $95{ }^{\circ} \mathrm{C}$ for $3 \mathrm{~min}, 30$ cycles of $95^{\circ} \mathrm{C}$ for $45 \mathrm{sec}, 57^{\circ} \mathrm{C}$ for $45 \mathrm{sec}$, and $72{ }^{\circ} \mathrm{C}$ for $2 \mathrm{~min}$. The final extension was $72{ }^{\circ} \mathrm{C}$ for $10 \mathrm{~min}$. The PCR products were electrophoresed in $1 \% \mathrm{w} / \mathrm{v}$ agarose gel.

The rice transformation protocol was based on Liu et al. (1998). About 50 dehusked Nipponbare grains were cleaned and washed before being placed on callus N6 agar supplemented with 2,4Dichlorophenoxyacetic acid (N6D2) (Huang et al., 2001) and grown in the dark at $28{ }^{\circ} \mathrm{C}$ for 2 weeks. The grown rice calli were then dissected into smaller sizes (2-3 $\mathrm{cm}$ ) and placed directly on a fresh N6D2 agar and further incubated in the dark at $28{ }^{\circ} \mathrm{C}$ for another 2 weeks. The transformed Agrobacterium was cultured in LB broth containing $50 \mu \mathrm{g} / \mathrm{mL}$ Kanamycin antibiotic at $28{ }^{\circ} \mathrm{C}$ for $19 \mathrm{~h}$ with agitation of $220 \mathrm{rpm}$. The culture was then grown in Agrobacterium minimal (AB) medium (Liu et al., 1998) with $50 \mu \mathrm{g} / \mathrm{mL}$ Kanamycin antibiotic at $28{ }^{\circ} \mathrm{C}$ for $4 \mathrm{~h}$. The culture was then centrifuged for $15 \mathrm{~min}$ at $4{ }^{\circ} \mathrm{C}$ at 3101 $x g$. The bacterial pellet was suspended in AAM induction medium (Hiei et al., 1997) with $200 \mu \mathrm{M}$ acetosyringone and cocultivated with the rice calli for $20 \mathrm{~min}$ at room temperature. The calli were then placed on N6D2-AS (Huang et al., 2001) agar layered with sterile Whatman No. 1 filter paper from Sigma-Aldrich (St. Louis, $\mathrm{MO})$. The calli were incubated in the dark at room temperature $\left(28{ }^{\circ} \mathrm{C}\right)$ for three days.
The rice transformant was selected three times with selection agar (Liu et al., 1998) with different dosages of hygromycin (25 $\mathrm{mg} / \mathrm{L}, \quad 50 \mathrm{mg} / \mathrm{L}$, and $50 \mathrm{mg} / \mathrm{L}$ respectively) and cefotaxime antibiotics (600 mg/L, $300 \mathrm{mg} / \mathrm{L}$, and $300 \mathrm{mg} / \mathrm{L}$, respectively). Survived rice transformants were grown on Murashige-Skoog Regeneration 1 (MSPR) agar (Liu et al., 1998) and incubated in the dark at room temperature $\left(28^{\circ} \mathrm{C}\right)$ for $7-8$ days. Next, they were placed in Murashige-Skoog Regeneration 2 (MSR) agar (Liu et al., 1998) to aid the root generation and incubated at room temperature with $12 \mathrm{~h}$ day light for 30-60 days. The transformant calli with growing root and shoot were placed in half strength of Murashige-Skoog (1/2 MS) agar (Liu et al., 1998) and further incubated for another 30 days. The transgenic seedlings were planted in Yangzhou University, Yangzhou, China until they matured. Genomic DNA was extracted from transgenic leaves using CTAB/chloroform method (Sambrook et al., 1989). The insertion of the transgene was confirmed by PCR using primers targeting the Hygromycin phosphotranspherase (HptII) gene $\mathrm{HygF}$ (5'-GGTCGCGGAGGCTATGGATGC-

3') and HygR (5'-
GCTTCTGCGGGCGATTTGTGT-3').

The PCR reaction was conducted in $30 \mu \mathrm{L}$ reaction with $15 \mu \mathrm{L} 2 \mathrm{X}$ Taq mastermix (Vazyme. China), $1.5 \mu \mathrm{L}(10 \mu \mathrm{M})$ of forward and reverse primers, and $25 \mathrm{ng}$ of plant genomic DNA. The amplification condition was as follows: $95{ }^{\circ} \mathrm{C}$ for $2 \mathrm{~min}$, 30 cycles of $95{ }^{\circ} \mathrm{C}$ for $30 \mathrm{sec}, 57{ }^{\circ} \mathrm{C}$ for $30 \mathrm{sec}$, and $72{ }^{\circ} \mathrm{C}$ for $1 \mathrm{~min}$ with the final extension of $72{ }^{\circ} \mathrm{C}$ for $10 \mathrm{~min}$. The PCR products were electrophoresed in $1 \% \mathrm{w} / \mathrm{v}$ agarose gel.

\subsection{GUS histochemical staining}

The leaf, stem, root, and grains of 35 DAP of T0 rice plants as well as leaf, root, 
and stem of 3 weeks old T1 rice seedling were collected and subjected to GUS staining procedure. GUS staining was performed using GUS histochemical assay kit from Real-Times (Beijing) Biotechnology (Beijing, China) according to the manufacturer's protocol. Untransformed Nipponbare was used as a negative control. The samples were examined with a dissecting microscope and photographed with Canon D1300 digital camera (Tokyo, Japan).

\subsection{GUS fluorometric assay}

The mature leaf, stem, and root samples of T0 transgenic rice were individually ground to a fine powder using liquid nitrogen. GUS fluorometry analysis was conducted as described by Jefferson et al. (1987). The 4-methylumbelliferyl b-Dglucuronide (4-MUG) was used as a substrate to quantify the GUS protein. Fourtime points were measured $(0 \mathrm{~min}, 20 \mathrm{~min}$, $40 \mathrm{~min}$, and $60 \mathrm{~min}$ ) with three replicates for each sample. Meanwhile, the total extract protein concentration of the transgenic rice plants was calculated using bovine serum albumin (BSA) as a standard protein. The calculation for determining the final protein concentration was conducted according to Bradford (1976). Spectrofluorometer was used to detect the fluorescence with the excitation and emission wavelength of $365 \mathrm{~nm}$ and 455 $\mathrm{nm}$, respectively. The GUS activity was expressed as pMole MUG release/min/mg protein.

\subsection{Statistical analysis}

The data for GUS activity was analysed using SAS system version 9.4. Two-way analysis of variance (ANOVA) was used and the means of GUS activity in different body parts of the same plants and the same body parts in different transgenic plants were compared using Tukey's test with a significant level of $(p<0.05)$.

\section{RESULTS AND DISCUSSIONS}

\subsection{Isolation and bioinformatic analysis of HvCslF6 putative promoter}

The $2771 \mathrm{bp}$ upstream of the TSS of Sloop HvCslF6 gene was successfully amplified, sequenced, and aligned with $H v C s l F 6$ promoter derived from seven other barley varieties as illustrated in Appendix 1. There were 16 variations identified including 12 single nucleotide polymorphisms (SNP), two insertions, and two substitutions. Insertion events of TTAG and TCTCTCAA were observed in all barley varieties except Sloop, Morex, CDCBold, and TR251 at the position between -1090 and -1435 from TSS. The alignment results demonstrated that there were less prominent differences in the $H v C s l F 6$ putative promoter among the barley varieties. The results were coherent with the previous study, which compared the HvCslF6 promoter of 35 barley genotypes (Garcia-Gimenez et al., 2019). Thus, the differences in the sequences of HvCslF6 promoter alone may not influence the characterisation and strength of the promoter regardless of the variety of barley used for investigation.

Based on the regulatory elements analysis of the promoter sequence using PlantCare and PLACE databases, multiple endosperm-specific regulating elements such as Dof, P-Box, E-box, CCAAT box, ACGT motif, AACA motif, and RY repeat were identified in the HvCslF6 putative promoter. The DNA-binding with one finger (DOF) motif (5'-AAAG-3') was the core sequence of prolamin box (5'CAAAAGG-3'). Both P-box and DOF motifs were responsible for the binding with protein, which activated the storage protein genes that mainly available in the cereal plant seed (Juhász et al., 2011). Furthermore, the presence of ACGT motif in the maize 22-kDA zein promoter was demonstrated to attract the maize opaque- 2 
transcription factor, which related to endosperm specific expression manner (Wang et al., 2013). The motif is also bound to other transcription factors required by the starch biosynthesis gene located in the maize grain (Wang et al., 2013). Moreover, CCAAT box is an enhancer that binds to the NF-Y transcription factor binding protein complex that is involved in seed development and embryo maturation of plants like Arabidopsis thaliana and soybean (Pelletier et al., 2017). The E box (5'-CAAACAC-3') is conserved in many storage protein gene promoters in cereal plants, indicating that it may direct expression in the cereal grain ( $\mathrm{Li}$ et al., 2019). The RY repeat targets B3 transcription factors, which is involved in the regulation of seed maturation of dicotyledonous plants such as soybean, broad bean, and Arabidopsis thaliana (Fauteux and Strömvik 2009). These endosperm-specific elements were scattered along the promoter, which more concentrated in less than $2771 \mathrm{bp}$ from the TSS of HvCslF6 gene (Figure 1). Hence, the study suggested that the optimum $H v C s l F 6$ promoter length for the strong expression of the GUS gene in the transgenic rice grain was less than $2771 \mathrm{bp}$.
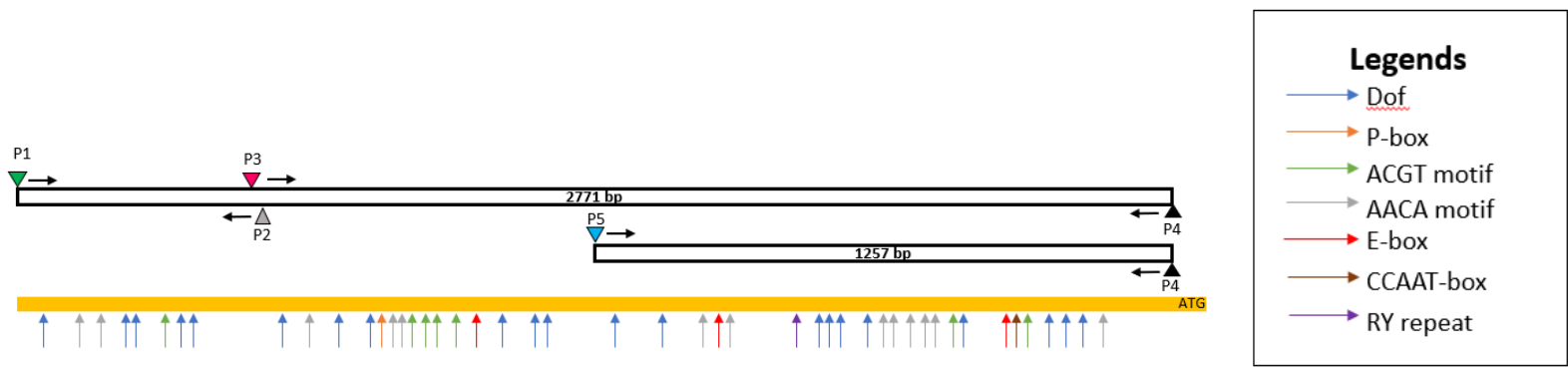

Figure 1. Amplification strategy for $2771 \mathrm{bp}$ and $1257 \mathrm{bp} H v C s l F 6$ promoter (above) and endosperm-specific motifs identified in $\mathrm{HvCslF6}$ promoter using PLACE and PLANTCARE databases (below).

\subsection{Development of transgenic rice for functional promoter analysis}

Two plant expression constructs were successfully assembled and transformed into Agrobacterium tumefaciens. This was confirmed using colony PCR of transformed Agrobacterium as depicted in Figure 2A and 2B. The bacteria were then co-cultivated with the
Nipponbare rice callus and grown until mature plants. The positive rice transformants were selected via amplification of HptII gene as portrayed in Figure 2C. Only three rice transformants were integrated with 2771 bp $H v C s l F 6$ prom::GUS, while two rice transformants contained 1257 bp $H v C s l F 6$ prom::GUS transgene. 


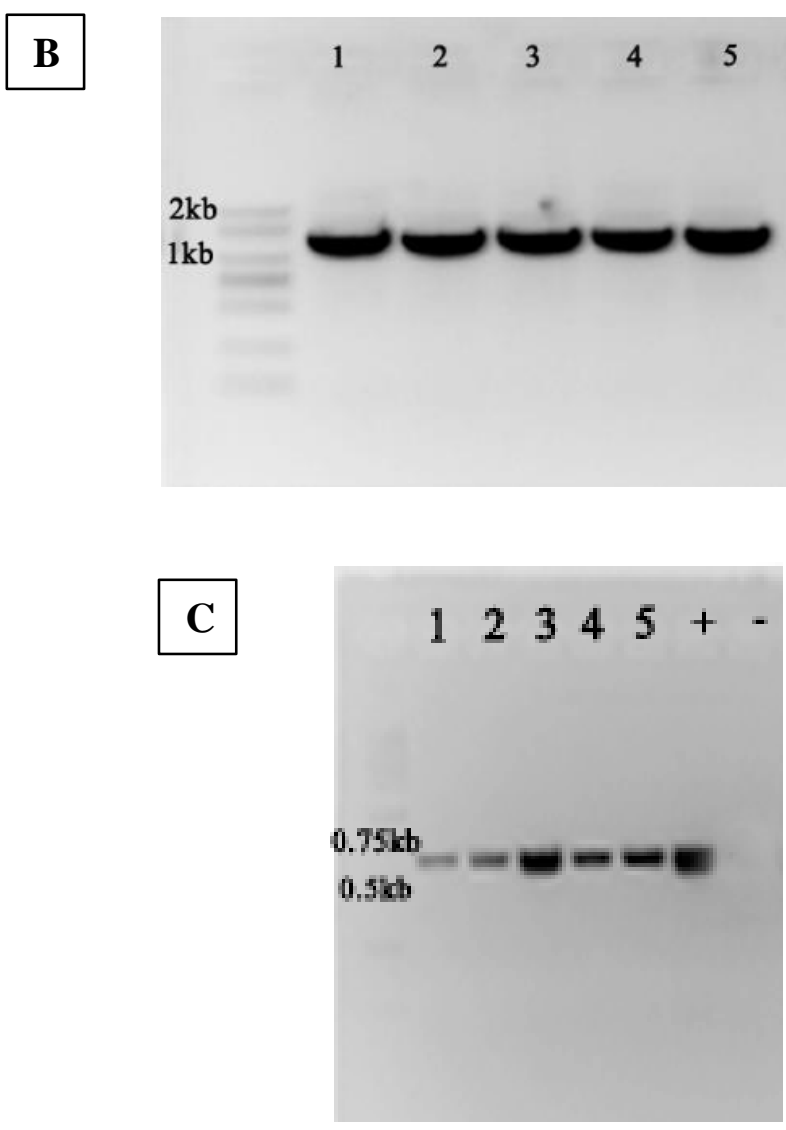

Figure 2. Regeneration of transgenic plants with interested construct. (A) Agarose gel of colony PCR Agrobacterium tumefaciens transformed with 2771 bp HvCslF6prom::GUS construct, M:5 kb DNA ladder, 1-5: potential positive colonies; (B) Agarose gel of colony PCR Agrobacterium tumefaciens transformed with 1257 bp HvCslF6prom::GUS construct, M: $2 \mathrm{~kb}$ DNA ladder, 1-5: potential positive colonies , +ve: positive control, -ve: negative control. ; (C) Agarose gel of amplification of HptII gene on transgenic rice. M: $2 \mathrm{~kb}$ DNA ladder, 1-3: Transgenic rice for 2771 bp HvCslF6prom::GUS construct 4-5: Transgenic rice for 1257 bp $H v C s l F 6$ prom::GUS construct, +: positive control

\subsection{Both HvCslF6 promoter lengths were functional in transgenic rice}

All mature T0 transgenic rice plants showed blue colour in multiple body parts after GUS histochemical staining procedure as depicted in Figure 3. Transgenic plants, regardless of the $\mathrm{HvCslF6}$ promoter lengths, were observed to express GUS protein moderately in the mid-section of the mature leaves while weakly expressed in the mature leaf tips. The blue GUS staining was also observed in the mature stem, root, and grain of the transgenic plants. In comparison to other body parts, an intense blue colour was found in the grain as the GUS expression was restricted to the embryo, endosperm tissues, and aleurone layer of the transgenic grain. The results agreed with the findings by previous studies, in which the HvCslF6 gene was expressed in the root, grain, stem, and mature leaf of barley while the highest expression was observed in the developing grain (Burton et al., 2006, 2008; Wong et al., 2015). Furthermore, the results also indicated that both promoters with different length shared a similar GUS expression pattern. 




Figure 3. Histochemical staining of the body parts of mature T0 transgenic rice.

Untransformed Nipponbare was used as a negative control. Scale bar $=1 \mathrm{~cm}$ 


\subsection{The HvCslF6 promoter strength was stable until T1 transgenic rice}

The $\mathrm{T} 1$ seedlings were grown until three weeks old before their body parts were subjected to GUS histochemical staining. The GUS protein was demonstrated to be expressed up to two generations of transgenic rice (Figure 4). This result was slightly similar to the overexpression of $\mathrm{HvCslF6}$ gene driven by strong constitutive $(C a M V 35 s)$ and endosperm-specific (Asglol) promoter in barley where the transgene expression was observed at $\mathrm{T} 0$ and the expression level increased at T1 generation (Burton et al., 2011). Based on Figure 4, the blue colour intensity of GUS staining was identified strongly in the roots of all transgenic T1 seedlings including small rootlets and root hair. In contrast, low intensity of blue staining was observed in the seedling leaf and stem.

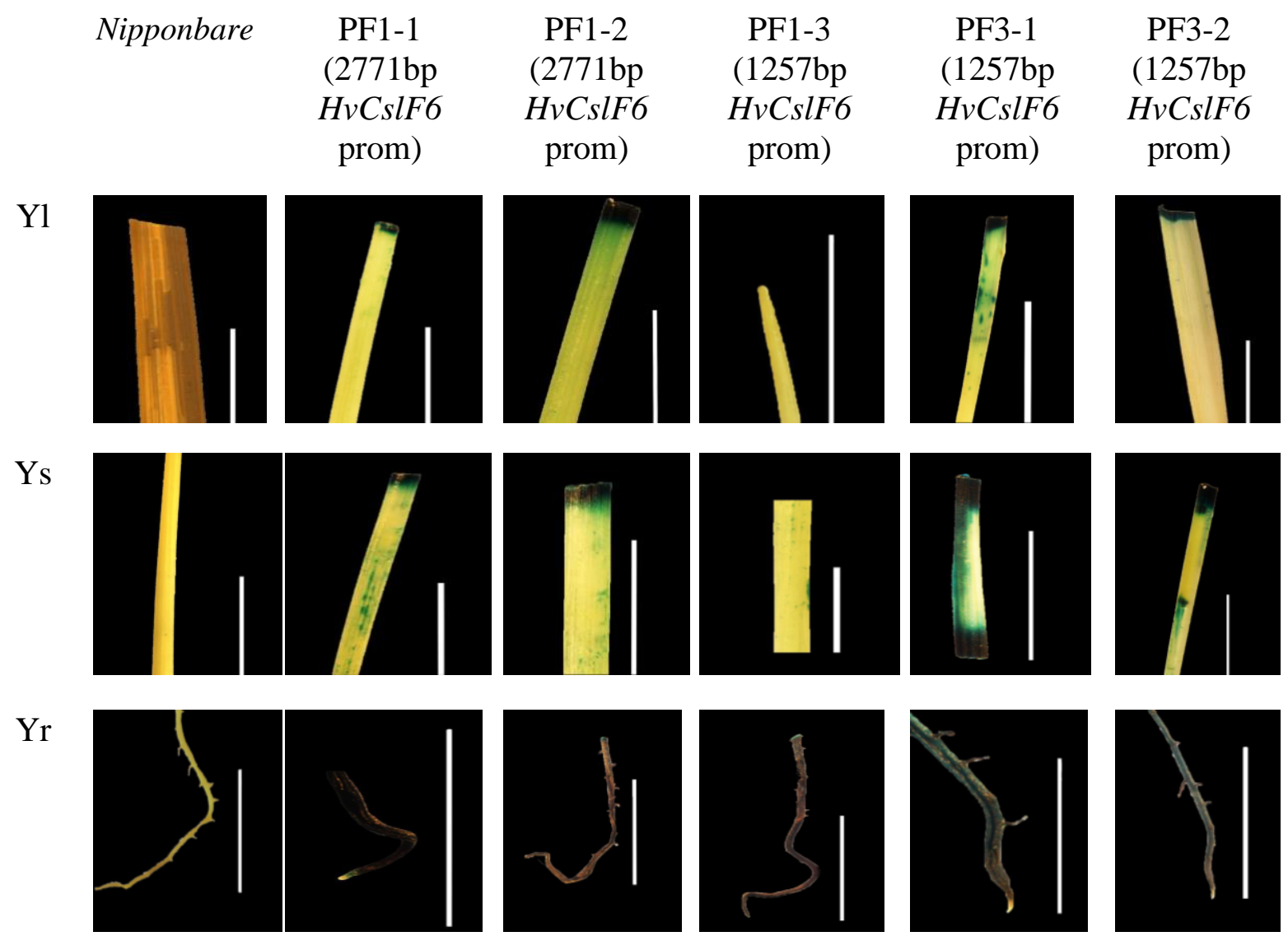

Figure 4. Histochemical staining of the body parts of $\mathrm{T} 1$ transgenic rice seedling. Untransformed Nipponbare was used as a negative control. Scale bar $=1 \mathrm{~cm}, Y 1$ : young leaf, Ys: Young stem, Yr: Young root

\subsection{The $1257 \mathrm{bp} \mathrm{HvCslF6}$ promoter drove stronger GUS expression in transgenic rice}

The GUS protein production profile driven by two different lengths of $H v C s l F 6$ promoter was compared via GUS histochemical staining and GUS quantitative assay. The GUS activity result was depicted in Figure 5. In the mature leaf, the GUS blue staining was most intense in the PZ3-1 with GUS activity of 41790 pmol 
4MU/min/mg protein. This was followed by PZ3-2 (26007 pmol 4MU/min/mg protein) and PZ1-1 (16715 pmol $4 \mathrm{MU} / \mathrm{min} / \mathrm{mg}$ protein). The least stained leaf was observed in PZ1-2 and PZ1-3 plants with GUS activity of 1981 pmol $4 \mathrm{MU} / \mathrm{min} / \mathrm{mg}$ protein and $2945 \mathrm{pmol}$ $4 \mathrm{MU} / \mathrm{min} / \mathrm{mg}$ protein respectively, which were not significantly different from each other but significantly different to that of the control. Moreover, there were no significant differences in the GUS activity of mature stem in PZ3-1 (25751 pmol 4MU/min/mg protein), PZ3-2 (23427 pmol $4 \mathrm{MU} / \mathrm{min} / \mathrm{mg}$ protein), and PZ1-1 (22011 pmol 4MU/min/mg protein). Similar to the GUS activity in mature leaves, PZ1-2 (6439 $\mathrm{pmol} 4 \mathrm{MU} / \mathrm{min} / \mathrm{mg}$ protein) and PZ1-3 (782 pmol $4 \mathrm{MU} / \mathrm{min} / \mathrm{mg}$ protein) also had the least GUS activity in the mature roots which were not significantly different compared to control. It is worthwhile to note that the GUS activity in the mature root of PZ1-1 was the highest (22889 $\mathrm{pmol} 4 \mathrm{MU} / \mathrm{min} / \mathrm{mg}$ protein), followed by PZ3-1 (15852 pmol 4MU/min/mg protein), PZ3-2 (12578 pmol 4MU/min/mg protein), PZ1-3 (9158 pmol 4MU/min/mg protein), and PZ1-2 (5838 pmol 4MU/min/mg protein). Based on the GUS activity for each transgenic plant, the PZ3-1 and PZ3-2 shared a slightly similar pattern while the transgene was not reactive in PZ1-1, PZ1-2, and PZ1-3 mature plants. The histochemical staining result matched the quantitative GUS activity results.

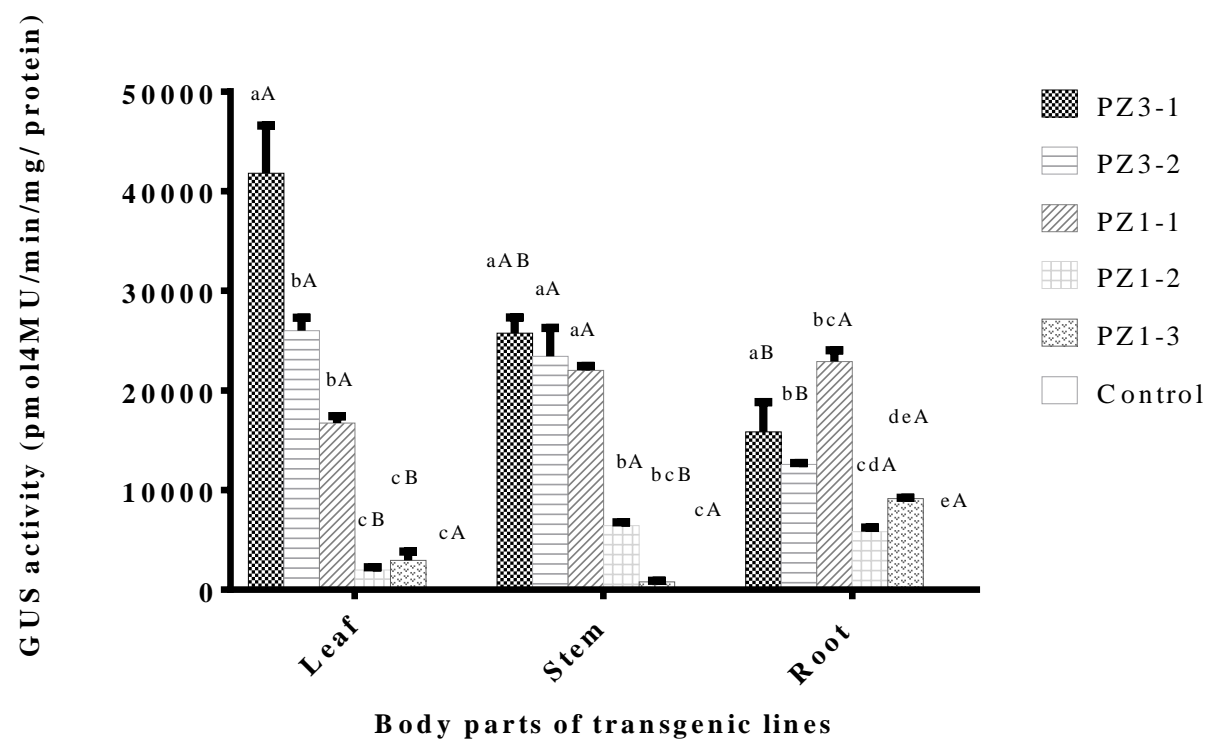

Figure 5. Graph of GUS activity T0 transgenic plants. PZ3-1 and PZ3-2 represent plants with 1257 bp HvCslF6 promoter while PZ1-1, PZ1-2, and PZ1-3 represent plants with 2771 bp HvCslF6 promoter. Non-transformed Nipponbare was used as a negative control. Twoway analysis of variance (ANOVA) and post hoc Tukey's test were used to determine the significant difference of GUS expression between each body part of each transgenic line. The small letter label demonstrates the significant differences in GUS activity of certain body part among the transgenic lines while the capital letter label shows the significant difference in GUS activity of the body parts of individual transgenic rice at $p<0.05$

There was no quantitative assay was performed on the transgenic grain of T0 generation. Thus, the GUS expression of transgenic grain analysis solely relied on the histochemical staining data. Based on the histochemical staining result of $\mathrm{T} 1$ seedling, the PZ1-1 seedling expressed a higher GUS production in the root but low 
in the leaf and stem. However, PZ3-1 showed moderate GUS staining in all body parts. It was also observed that lower GUS protein accumulation was observed in all three body parts of PZ1-2 and PZ1-3 as compared to the PZ3-1, PZ3-2, and PZ1-1. Overall, it was suggested that PZ3-1 and PZ3-2 plants showed higher GUS expression in comparison to PZ1-1, PZ1-2, and PZ1-3. There was a significance different in the GUS activity within the individual plants containing the same construct. In overall body parts, PZ3-1 showed double GUS activity than PZ3-2 while PZ1-1 expressed 3-5 folds activity in comparison to PZ1-2 and PZ1-3 plants. The difference in GUS activity may be due to the differences in $G U S$ gene copy number in each plant, which may yield a different amount of expressed protein. This will affect the gene activity in the transgenic plants (Hobbs et al., 1990). Thus, determination of gene copy number of individual plants is required by identifying the amount of GUS gene with the reference of rice endogenous sucrose phosphatase gene following the procedure developed by Ding et al. (2004).

Overall, the truncated promoter generated a stronger GUS expression in all body parts of the plant in comparison to the 2771 bp HvCslF6 promoter. One of the possible reasons for this observation was the presence of repression elements in the region of $1257 \mathrm{bp}$ to $2771 \mathrm{bp}$ upstream from HvCslF6 gene. Dimitroff (2016) suggested that there was a repressor element in between the $1750 \mathrm{bp}$ and $2500 \mathrm{bp}$ upstream region of HvCslF6 promoter. The suggestion was based on the lower expression of the Luciferase $(L u c)$ gene in the truncated $2.5 \mathrm{~kb}$ and $2.25 \mathrm{~kb}$ HvCslF6prom::Luc gene constructs while higher expression when truncated $1.75 \mathrm{~kb}$ HvCslF6prom::Luciferase gene constructs were tested. Based on this study, it can be concluded that the 1257 bp HvCslF6 promoter length has the functional promoter length to drive the expression of the GUS gene in the transgenic rice. However, the shorter length of the promoter $(0.25-1 \mathrm{~kb})$ can be further investigated as they were reported to have stronger expression in transgenic barley (Dimitroff 2016).

\section{CONCLUSIONS}

This study reported a promising expression construct of $\mathrm{HvCslF6}$ promoter to drive reporter gene in transgenic rice. Multiple endosperm-specific motifs identified in the $\mathrm{HvCslFG}$ promoter region might be responsible for the endospermspecific promoter activity pattern. Two expression constructs were developed consisted of $2771 \mathrm{bp}$ and $1257 \mathrm{bp}$ region upstream to HvCslF6 gene, then fused with GUS gene and introduced into Nipponbare rice cultivar. The integration of the foreign DNA into the rice genome did not adversely affect the general development of the rice plant. The histochemical staining of transgenic rice containing 2771 bp HvCslF6prom::GUS showed blue GUS staining in the leaf, stem, root, and seed of plants. The truncated $1257 \mathrm{bp}$ HvCslF6prom::GUS expressed a slightly similar spatial pattern with the $2771 \mathrm{bp}$ length based on the GUS histochemical analysis. The GUS fluorometry results summarized that the 1257 bp $H v C s l F 6$ prom::GUS generated a stronger GUS expression in all body parts in comparison to the $2771 \mathrm{bp}$ HvCslF6prom::GUS. The activities of both expression constructs remained in the $\mathrm{T} 1$ seedlings indicated that they were stable up until the second generation of transgenic plants. Therefore, the $1257 \mathrm{bp} \mathrm{HvCslF6}$ promoter length has the functional promoter length to drive the expression of the GUS gene in the transgenic rice. Regardless of their length, both expression constructs of $\mathrm{HvCslF6}$ promoter showed a promising alternative to the frequently used 
rice Glutelin $1(G t 1)$ promoter in expressing transgene in plants based on their strong expression specifically in the seed of the host plants.

\section{ACKNOWLEDGEMENT}

The authors gratefully acknowledge the infrastructure and support of Universiti Putra Malaysia Bintulu Sarawak Campus, Malaysia and Key Laboratory of Plant Functional Genomics, Yangzhou University, China. This project is funded by Ministry of Higher Education Malaysia Fundamental Research Grant Scheme (FRGS/1/2015/SG05/UPM/03/3), (Project No.:02-01-15-1761FR).

\section{REFERENCES}

Arena, M. P., Graziano C., Fiocco D., Russo, P., Torelli, M., Spano, G., Capozzi, V. (2014) Barley $\beta$ Glucans-Containing Food Enhances Probiotic Performances of Beneficial Bacteria, International Journal of Molecular Sciences 15 (2): 3025-3039.

Bradford, Marion M. 1976. "A Rapid and Sensitive Method for the Quantitation of Microgram Quantities of Protein Utilizing the Principle of Protein-Dye Binding." Analytical Biochemistry 72 (1): 248-54.

Burton, R. A., Collins, H.M., Kibble, N. A. J., Smith, J. A., Shirley, N. J., Jobling, S. A., Henderson, M. (2011) Over-Expression of Specific HvCslF Cellulose Synthase-like Genes in Transgenic Barley Increases the Levels of Cell Wall $(1,3 ; 1,4)-\beta$-D-Glucans and Alters Their Fine Structure, Plant Biotechnology Journal 9 (2): 117135.
Burton, R. A., Jobling, S. A., Harvey, A. J., Shirley, N. J., Mather, D. E., Bacic, A., Fincher, G. B. (2008) The Genetics and Transcriptional Profiles of the Cellulose SynthaseLike HvCslF Gene Family in Barley, Plant Physiology 146 (4): 1821-33.

Burton, R. A., Wilson, S. M., Hrmova, M., Harvey, A. J., Shirley, N. J., Medhurst, A., Stone, B. A., Newbigin, E. J., Bacic, A., Fincher, G. B. (2006) Cellulose Synthaselike CslF Genes Mediate the Synthesis of Cell Wall $(1,3 ; 1,4)$ Beta-D-Glucans, Science 311 (5769): 1940-1942.

Dimitroff, G. (2016) Investigating the Synthesis and Regulation of $(1,3 ; 1,4)$-Beta-Glucan

Biosynthesis, Australia: University of Adelaide.

Ding, J., Jia, J., Yang, L., Wen, H., Zhang, C., Liu, W., Zhang, D. (2004) Validation of a Rice Specific Gene, Sucrose Phosphate Synthase, Used as the Endogenous Reference Gene for Qualitative and Real-Time Quantitative PCR Detection of Transgenes, Journal of Agricultural and Food Chemistry 52 (11): 337277.

Ermawar, R. A., Collin, H. M., Byrt, C. S., Henderson, M., O’Donovan, L. A., Shirley, N. J., Schwerdt, J. G., Lahnstein, J., Fincher, G. B., Burton R.A. (2015) Genetics and Physiology of Cell Wall Polysaccharides in the Model C4 Grass, Setaria Viridis Spp, BMC Plant Biology 15 (236)

Fu, C., Donovan, W. P., ShikapwashyaHasser, O., Ye, X., Cole, R. H. (2014) Hot Fusion: An Efficient Method to Clone Multiple DNA Fragments as Well as Inverted Repeats without Ligase, PLOS ONE 9 (12): e115318 
Garcia-Gimenez, G., Russell, J., Aubert, M. K., Fincher, G. B., Burton, R. A., Waugh, R., Tucker, M. R., Houston, K. (2019) Barley Grain $(1,3 ; 1,4)-\beta$ Glucan Content: Effects of Transcript and Sequence Variation in Genes Encoding the Corresponding Synthase and Endohydrolase Enzymes, Scientific Reports 9 (1): 1-11.

Joyce, S. A., Kamil, A., Fleige, L., Gahan, C. G. M. (2019) The CholesterolLowering Effect of Oats and Oat Beta Glucan: Modes of Action and Potential Role of Bile Acids and the Microbiome, Nutrition, 171

Juhász, A., Makai, S.,Sebestyén, E., Tamás, L., Balázs, E. (2011). Role of Conserved Non-Coding Regulatory Elements in LMW Glutenin Gene Expression, PLOS ONE 6 (12): e29501

Higo, K., Ugawa, Y., Iwamoto, M., Higo, H. (1998) PLACE: A Database of Plant Cis -Acting Regulatory DNA Elements, Nucleic Acids Research 26 (1): 358-59.

Hiei, Yukoh, Toshihiko Komari, and Tomoaki Kubo. 1997. "Transformation of Rice Mediated by Agrobacterium Tumefaciens." Plant Molecular Biology 35 (1-2): 205-18.

Hobbs, S. L., Kpodar, P., DeLong. C. M. (1990) The Effect of T-DNA Copy Number, Position and Methylation on Reporter Gene Expression in Tobacco Transformants, Plant Molecular Biology 15 (6): 851-64.

Huang, J., Wei, Z., An, H., \& Zhu, Y. (2001). Agrobacterium tumefaciens -mediated transformation of rice with the spider insecticidal gene conferring resistance to leaf folder and striped stem borer. Cell Research, 11, 149-155.

Kim, S., Zemelis-Durfee, S., Jensen, J. K., Wilkerson, C.G., Keegstra, K.,
Brandizzi, F. (2018) In the Grass Species Brachypodium Distachyon, the Production of Mixed-Linkage $(1,3 ; 1,4)-\beta$-Glucan (MLG) Occurs in the Golgi Apparatus, The Plant Journal 93 (6): 1062-1075.

Lescot, M., Déhais, P., Thijs, G., Marchal, K., Moreau, Y., Peer, Y. V. D., Rouzé, P., Rombauts, S. (2002) PlantCARE, a Database of Plant Cis-Acting Regulatory Elements and a Portal to Tools for in Silico Analysis of Promoter Sequences, Nucleic Acids Research 30 (1): 325-27.

Lim, W. L., Collin, H. M., Byrt, C. S., Lahnstein, J., Shirley, N. J., Aubert, M. K., Tucker, M. R., Peukert, M., Matros, A., Burton, R. A. (2019) Overexpression of HvCsiF6 in Barley Grain Alters Carbohydrate Partitioning plus Transfer Tissue and Endosperm Development, Journal of Experimental Botany 71 (1): 138-53.

Li, S., Chen, L., Peng, X., Wang, C., Qin, B., Tan, D., Han, C. (2019) Overview of the Reporter Genes and Reporter Mouse Models, Animal Models and Experimental Medicine, (8) 29-35.

Little, A., Schwerdt, J., Shirley, N. J., Khor, S. F., Neumann, K., O’Donovan, L. A., Lahnstein, J. (2018) Revised Phylogeny of the Cellulose Synthase Gene Superfamily: Insights into Cell Wall Evolution, Plant Physiology 177 (7): 11241141.

Liu, Q., Zhang J., Wang, Z., Hong, M., Gu, M. (1998) A highly efficient transformation system mediated by Agrobacterium tumefaciens in rice ( Oryza sativa L.), Acta Phytophysiol Sin 24 (3): 259-71.

Marković, S. M.,Đukić N. H., Knežević, D., Leković, S. V. (2017) Divergence of Barley and Oat 
Varieties according to the Content of $\quad \beta$-Glucan (Short Communication), Journal of the Serbian Chemical Society 82 (4): 379-88.

Pelletier, J. M., Kwong, R. W., Park, S., Le, B. H., Baden, R., Cagliari, A., Hashimoto, M. (2017) LEC1

Sequentially Regulates the Transcription of Genes Involved in Diverse Developmental Processes during Seed Development, Proceedings of the National Academy of Sciences 114 (32): E6710-19.

Ren, Y., Xie, H, Liu, L., Jia, D., Yao, K., Chi, Y. (2018) Processing and Prebiotics Characteristics of $\beta$ Glucan Extract from Highland Barley, Applied Sciences 8 (1481): $1-11$.

Wang, J. C., Xu, H., Zhu, Y., Liu, Q. Q., Cai, X. L. (2013) OsbZIP58, a Basic Leucine Zipper Transcription Factor, Regulates Starch Biosynthesis in Rice Endosperm, Journal of Experimental Botany 64 (11): 3453-3466.

Wilson, S. M., Ho, Y. Y., Lampugnani, E. R., Van de Meene a. M. L., Bain, M. P., Bacic, A., Doblin, M.S. (2015) Determining the Subcellular Location of Synthesis and Assembly of the Cell Wall Polysaccharide $\quad(1,3 ; 1,4)-\beta$-DGlucan in Grasses, The Plant Cell 27 (3): 754-71.
Wolever, T., Tosh, S., Gibbs, A., BrandMiller, J., Duncan, A., Hart, V., Lamarche, B., Thomson, B., Duss, R., Wood, P. (2010) Physicochemical Properties of Oat Beta-Glucan Influence Its Ability to Reduce Serum LDL Cholesterol in Humans: A Randomized Clinical Trial, The American Journal of Clinical Nutrition 92 (4): 723-32.

Wong, S. C., Shirley, N. J., Little, A., Khoo, K. H. P., Schwerdt, J., Fincher, G. B., Burton, R. A., Mather, D. E. (2015) Differential Expression of the HvCslF6 Gene Late in Grain Development May Explain Quantitative Differences in $(1,3 ; 1,4)-\beta$-Glucan Concentration in Barley, Molecular Breeding 35 (1): $1-12$.

Sambrook, J., Fritsch, E. F., Maniatis, T. (1989). Molecular Cloning: A Laboratory Manual. 2nd ed. Vol. 1, New York: Cold Spring Harbor Laboratory Press.

Vega-Sánchez, M. E., Verhertbruggen, Y., Christensen, U., Chen, X., Sharma, V., Varanasi, P., Jobling, S. A. (2012) Loss of Cellulose SynthaseLike F6 Function Affects MixedLinkage Glucan Deposition, Cell Wall Mechanical Properties, and Defense Responses in Vegetative Tissues of Rice, Plant Physiology 159 (1): 56-69. 


\section{APPENDIX}

Appendix 1: Alignment of $H v C s l F 6$ putative promoter region of Sloop against other barley variety using Geneious 9.0 software

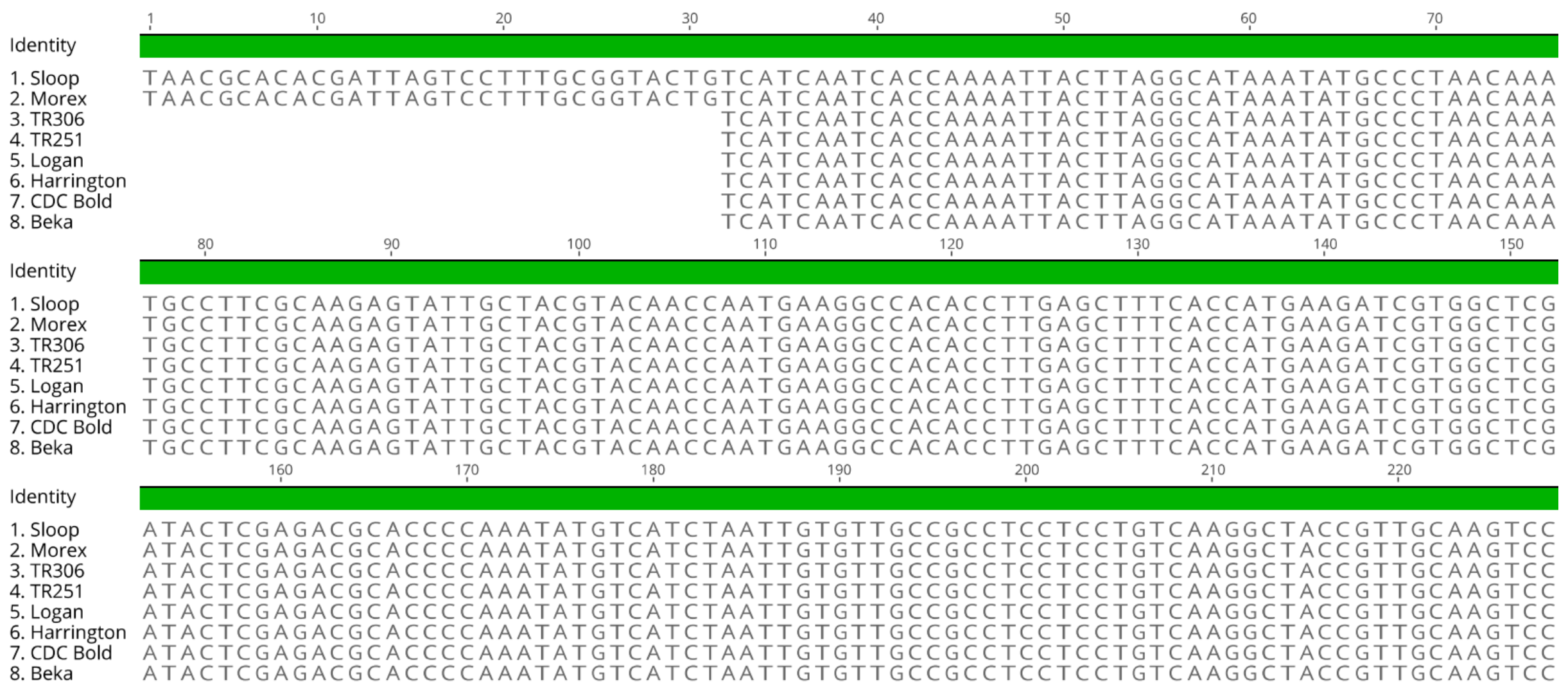


Identity

1. Sloop

2. Morex

3. TR306

4. TR251

5. Logan

6. Harrington

8. Beka

Identity

1. Sloop

2. Morex

3. TR306

4. TR251

5. Logrington

7. CDC Bold

8. Beka

Identity

1. Sloop

2. Morex

3. TR306

4. TR251

5. Logan

6. Harringto

8. Beka

Identity

1. Sloop

2. Morex

3. TR306

4. TR251

5. Logan

6. Harrington

7. CDC Bold

8. Beka

TCAACACTCGACACAGAGGAAAAACCCTGTGCCACATGAGCGAGTTGAATCCAAAACCTCTAGCAGCAAGAATCTC TCAACACTCGACACAGAGGAAAAACCCTGTGCCACATGAGCGAGTTGAATCCAAAACCTCTAGCAGCAAGAATCTC TCAACACTCGACACAGAGGAAAAACCCTGTGCCACATGAGCGAGTTGAATCCAAAACCTCTAGCAGCAAGAATCTC TCAACACTCGACACAGAGGAAAAACCCTGTGCCACATGAGCGAGTTGAATCCAAAACCTCTAGCAGCAAGAATCTC TCAACACTCGACACAGAGGAAAAACCCTGTGCCACATGAGCGAGTTGAATCCAAAACCTCTAGCAGCAAGAATCTC TCAACACTCGACACAGAGGAAAAACCCTGTGCCACATGAGCGAGTTGAATCCAAAACCTCTAGCAGCAAGAATCTC TCAACACTCGACACAGAGGAAAAACCCTGTGCCACATGAGCGAGTTGAATCCAAAACCTCTAGCAGCAAGAATCTC 320 330 360

CATAATAGTTTTGTGCTCAATCATGTCAATTACTTTTTCAAAGTCTTGACTTGAGAACAATAAATTCCTCTCTAGA

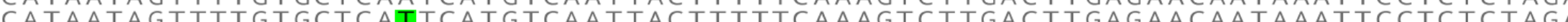
作 CATAATAGTTTTGTGCTCAATCATGTCAATTACTTTTTCAAAGTCTTGACTTGAGAATAATAAATTCCTCTCTAGA CATAATAGTTTTGTGCTCAATCATGTCAATTACTTTTTCAAAGTCTTGACTTGAGAACAATAAATTCCTCTCTAGA CATAATAGTTTTGCTCAATCATG CAATATA


420 430 450 CTGGTGGCATTGATGGATGAATTCATATGCCCAGATCATAGAATCTTGGATGGATCTCTTTATAAGAAAACCATAT CTGGTGGCATTGATGGATGAATTCATATGCCCAGATCATAGAATCTTGGATGGATCTCTTTATAAGAAAACCATAT 作 GTGGTGGCATTGTGTGATGAATTCATATGCCCAGATCATAGAATCTTGGATGGATCTCTTTATAAGAAAACCATAT CTGGTGGCATTGATGGATGAATTCATATGCCCAGATCATAGAATCTTGGATGGATCTCTTTATAAGAAAACCATAT CTGGTGGCATTGATGGATGAATTCATATGCCCAGATCATAGAATCTTGGATGGATCTCTTTATAAGAAAACCATATATATATA CTGGTGGCATTGATGGATGAATTCATATGCCCAGATCATAGAATCTTGGATGGATCTCTTTATAAGAAAACCATAT TATGAATTCATATGCCCAGATCATAGAATCTTGGATGGATCTCTOTATAAGAAAACCATAT 470 480 490 510 520

TGATTTGTGTGAATCATACTACTACCTCCGTCCCGGTGTATAAGTCATTTGCGTAGTTCTAGGTCATCGATTTGAG TGATTTGGTGATCATACTACTACCTCCGTCCCGTGTATAAGTATTTGCGTATTCTAGTCATCGATTTGG TGATTTGTGTAATCATACTACTACCTCCGTCCCGGTGTATAAGTCATTTGCGTAGTTVTAGGTCATCGATTTGAG TGATTTGTGTGAATCATACTACTACCTTCGTCCCGGTGTATAAGTCATTTGCGTAGTTCTAGGTCATTGATTTGAG TGATTTGTGTGAATCATACTACTACCTCCGTCCCGGTGTATAAGTCATTTGCGTAGTTCTAGGTCATCGATTTGAG TGATTTGTGTGAATCATACTACTACCTCCGTCCCGGTGTATAAGTCATTTGCGTAGTTVTAGGTCATCGATTTGAG TGATTTGTGTGAATCATACTACTACCTCCGTCCCGGTGTATAAGTCATTTGCGTAGTTCTAGGTCATCGATTTGAG 
Identity

1. Sloop

2. Morex

3. TR306

4. TR251

5. Logan

6. Harrington

AATTAAATATGTGTTATATGTCATAAAAAGTATATCATTAGATTTCTACATAGATGTAGTTTCTAAATTTATATT TAATTAAATATGTGTTATATGTCATAAAAAGTATATCATTAGATTTCTACATAGATGTAGTTTCTAAATTTATATT TAATTAAATATGTGTTATATGTCATAAAAAGTATATCATTAGATTTCTACATAGATGTAGTTTCTAAATTTATATT TAATTAAATATGTGTTATATGTCATAAAAAGTATATCATTAGATTTCTACATAGATGTAGTTTCTAAATTTATATT TAATTAAATATGTGTTATATGTCATAAAAAGTATATCATTAGATTTCTACATAGATGTAGTTTCTAAATTTATATT

CDC Bold 作 TAATTAAATATGTGTTATATGTCATAAAAAGTATATCATTAGATTTCTACATAGATGTAGTTTCTAAATTTATATT 610 680

Identity

1. Sloop 2. Morex 3. TR306 4. TR251 5. Logan 6. Harrington

TTTGTTACATATGATACATATTTAGATAGTTAAATTGTCGACCTAGAACTACGTGAAAGACTTATACACCGGGAC

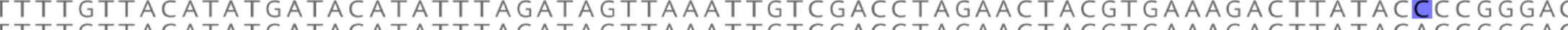

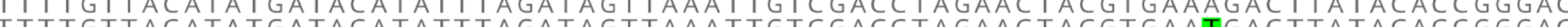


TTTGTTACATATGATACATATTTAGATAGTTAAATTGTCGACCTAGAACTACGTGAAAGACTTATACACCGGGAC TITTACATATGATACATATTTAGATAGTTAAATTGTCGACCTAGAACTACGTGAAAGACTTATACGCCGGGAC


690 700 710 720 730

GGAGGGAGTACCTTCTTTTCGGTTTATAAGGCGTGCACATATCTTTAGGTTGAAAAATAGACCAACTTAATACGAG GGAGGGAGTACCTTCTTTTCGGTTTATAAGGCGTGCACATATCTTTAGGTTGAAAAATAGACCAACTTAATACGAG GGAGGGAGTACCTTCTTTTCGGTTTATAAGGCGTGCACATATCTTTAGGTTGAAAAATAGACCAACTTAATACGAG

. TR251 GGAGGGAGTACCTTCTTTTCGGTTTATAAGGCGTGCACGTATCTTTAGGTTGAAAAATAGACCAACTTAATACGAG GGAGGGAGTACCTTCTTTTCGGTTTATAAGGCGTGCACATATCTTTAGGTTGAAAAATAGACCAACTTAATACGAG

7. CDCBold GGAGGGAGTACCTTCTTTTCGGTTTATAAGGCGTGCACATATCTTTAGGTTGAAAAATAGACCAACTTAATACGAG 8. Beka GGAGGGAGTACСTTCTTTTCGGTTTATAAGGCGTGCACATATCTTTAGGTTGAAAAATAGACCAACTTAATACGAG 
dentity

1. Sloop

2. Morex

3. TR306

4. TR251

5. Logan

6. Harrington
7. CDC Bold

8. Beka

Identity

1. Sloop

2. Morex

3. TR306

4. TR251

5. Logan

6. Harrington

7. CDC Bol

8. Beka

Identity

1. Sloop

2. Morex

3. TR306

4. TR251

5. Logan

6. Harrington

7. CDC Bold

8. Beka

Identity

1. Sloop

2. Morex

3. TR306

4. TR251

5. Logan

6. Harringto

7. CDC Bold

8. Beka

TTATATATCATTGAAAATTTCAAATCGTCTATTTTCTAATGATATAATTATTAGACTATACGGCTCAACTTATATT TTATATATCATTGAAAATTTCAAATCGTCTATTTTCTAATGATATAATTATTAGACTATACGGCTCAACTTATATT TATATATCATTGAAAATTTCAAATCGTCTATTTTCTAATGATATAATTATTAGACTATACGGCTCAACTTATATT TTATATATCATTGAAAATTTCAAATCGTCTATTTTCTAATGATATAATTATTAGACTATACGGCTCAACTTATATT TTATATATCATTGAAAATTTCAAATCGTCTATTTTCTAATGATATAATTATTAGACTATACGGCTCAACTTATATT TTATTATCATTGAAAATTTCAAATCGTCTATTTTCTAATGATATAATTATTAGACTATACGGCTCAACTTATATT TTATATATCATTGAAAATTTCAAATCGTCTATTTTCTAATGATATAATTATTAGACTATACGGCTCAACTTATATT TTATATATCATTGAAAATTTCAAATCGTCTATTTTCTAATGATATAATTATTAGACTATACGGCTCAACTTATATT 840 
Identity

1. Sloop

2. Morex

3. TR306

4. TR251

6. Harrington

8. Beka

Identity

1. Sloop

2. Morex

3. TR306

4. TR251

5. Logan

6. Harrington
7. CDC Bold

8. Beka

Identity

1. Sloop

2. Morex

3. TR306

4. TR251

5. Logan

6. Harrington

7. CDC Bold

8. Beka

Identity

1. Sloop

2. Morex

3. TR306

4. TR251

5. Logan

7. CDC Bold

7. CDC Bold
8. Beka

TGTCCACACATAGACGCGCGCGCACACATAAGCAAGTGAGTAGTAGGTGATTACATTACCGTCGGCGTGAAGCGTT TGTCCACACATAGACGCGCGCGCACACATAAGCAAGTGAGTAGTAGGTGATTACATTACCGTCGGCGTGAAGCGTT

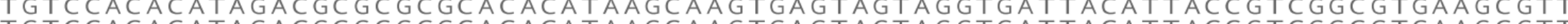

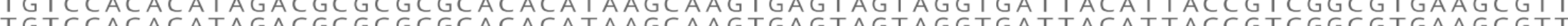


TGTCCACACATAGACGCGCGCGCACACATAAGCAAGTGAGTAGTAGGTGATTACATTACCGTCGGCGTGAAGCGTT TGTC CACACATAGACGCGCGCGCACACATAAGCAAGTGAGTAGTAGGTGATTACATTACCGTCGGCGTGAAGCGTT TGTCCACACATAGACGCGCGCGCACACATAAGCAAGTGAGTAGTAGGTGATTACATTACCGTCGGCGTGAAGCGTT 1,150 1,160 1,170 1,180 1,190 1,200 1,210

AAAATTGCGTCCGTTCTCTCCCGCCTCCTCGTAAATGCTTTGGGACTCGTTGATTGTAGCAGTGGTAGTTTATCAT 作

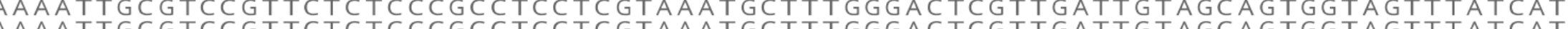



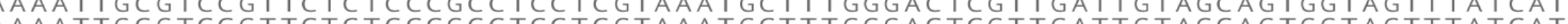


AAAATTGCGTCCGTTCTCTCCCGCCTCCTCGTAAATGCTTTGGGACTCGTTGATTGAGCAGTGGTAGTTTATCAT AAAATTGCGTCCGTTCTCTCCCGCCTCCTCGTAAATGCTTTGGGACTCGTTGATTGTAGCAGTGGTAGTTTATCAT $1,220 \quad 1,230$ 1,240 1,250 1,260 1,270 1,280 1,290

GAATGCTGGGGCTAGCGTG-CCCGCATATGTAGTTGGATCGTCGATGCAACAGCAAGGCCGGTTAATTACTCCTCC GAATGCTGGGGCTAGCGTG-CCCGCATATGTAGTTGGATCGTCGATGCAACAGCAAGGCCGGTTAATTACTCCTCC GAATGCTGGGGCTAGCGTGCCCCGCATATGTAGTTGGATCGTCGATGCAACAGCAAGGCCGGTTAATTACTCCTCC GAATGCTGGGGCTAGCGTGCCCCGCATATGTAGTTGGATCGTCGATGCAACAGCAAGGCCGGTTAATTACTCCTCC GAATGCTGGGGCTAGCGTGCCCCGCATATGTAGTTGGATCGTCGATGCAACAGCAAGGCCGGTTAATTACTCCTCC GAATGCTGGGGCTAGCGTGCCCCGCATATGTAGTTGGATCGTCGATGCAACAGCAAGGCCGGTTAATTACTCCTCC GAATGCTGGGGCTAGCGTGCCCCGCATATGTAGTTGGATCGTCGATGCAACAGCAAGGCCGGTTAATTACTCCTCC GAATGCTGGGGCTAGCGTGCCCCGCATATGTAGTTGGATCGTCGATGCAACAGCAAGGCCGGTTAATTACTCCTCC 1,300 1,310 1,320 1,330 1,340 1,350 1,360

GGCCTCTCCATAC CAGTGCACGGCTCGCCACGATCGTTTTCTTGTGGTATGTTGTCCTTTTGTGTGTTGTGTGGCG GGCCTCTCCATACCAGTGCACGGCTCGCCACGATCGTTTTCTTGTGGTATGTTGTCCTTTTGTGTGTTGTGTGGCG GGCCTCTCCATACCAGTGCACG CTCGCCACGATCGTTTTCTTGTGGTATGTTGTCCTTTTGTGTGTTGTGTGGCG GGCCTCTCCATACCAGTGCACGGCTCGCCACGATCGTTTTCTTGTGGTATGTTGTCCTTTTGTGTGTTGTGTGGCG G GGCCTCTCCATACCAGTGCACGGCTCGCCACGATCGTTTTCTTGTGGTATGTTGTCCTTTTGTGTGTTGTGTGGCG GGCCTCTCCATACCAGTGCACGGCTCGCCACGATCGTTTTCTTGTGGTATGTTGTCCTTTTGTGTGTTGTGTGGCG 


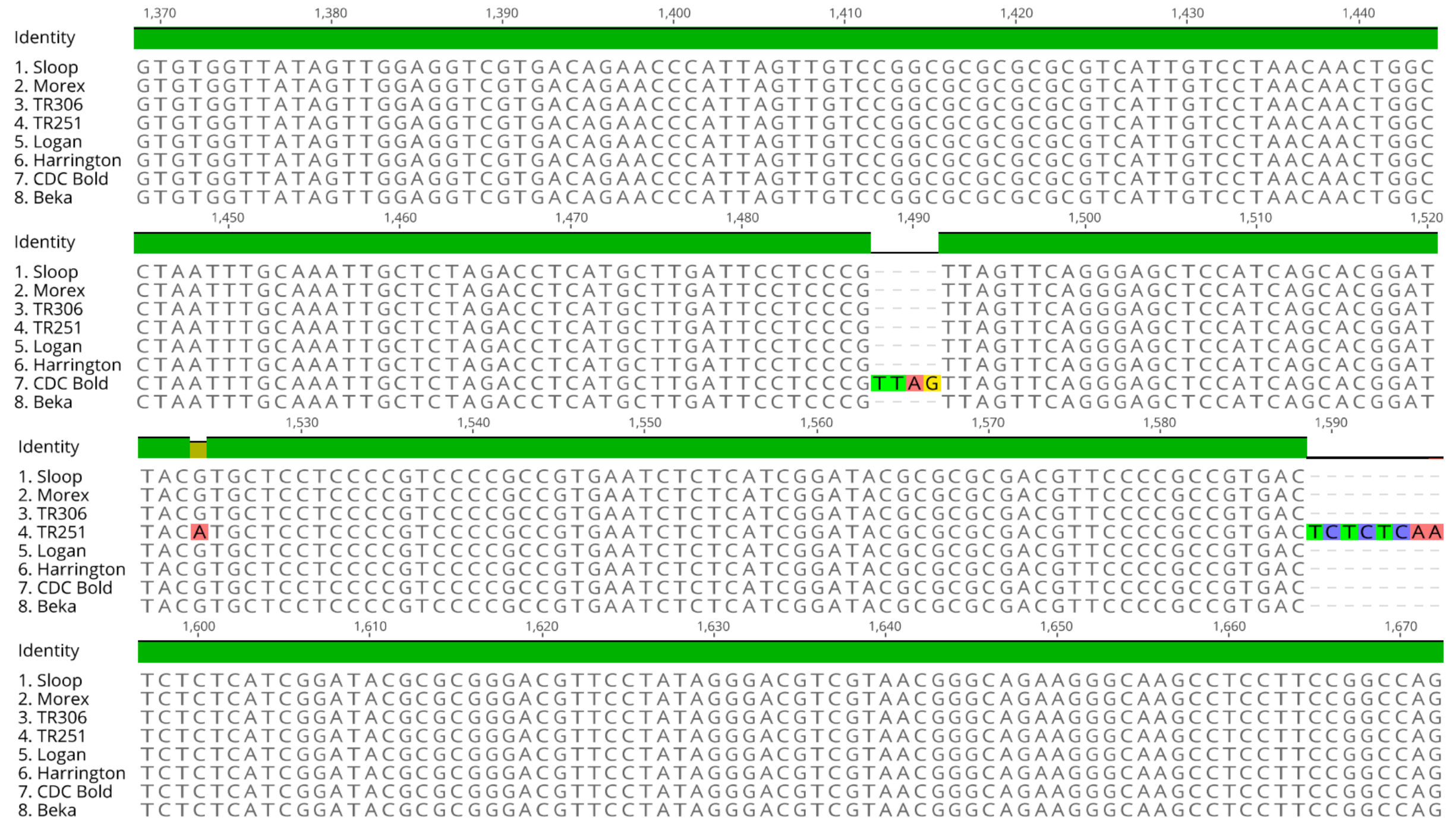


Identity

1. Sloop

2. Morex

3. TR306

4. TR251

5. Logan

6. Harrington

7. CDC Bold

8. Bek

Identity

1. Sloop

2. Morex

3. TR30

4. TR251

5. Logan

6. Harrington

8. Beka

Identity

1. Sloop

2. Morex

3. TR306

4. TR25

5. Logan

6. Harrington
7. CDC Bold

8. Beka

Identity

1. Sloop

2. Morex

3. TR306

4. TR251

5. Logan

7. CDC Bold

8. Beka

GGGACCAATGCATTCCTTCTCGTGAAGCATTTGCTCGCCAAAGCCAAAGCCAAAGCCAAAGCCACAAGGGGAAAAA GGGACCAATGCATTCCTTCTCGTGAAGCATTTGCTCGCCAAAGCCAAAGCCAAAGCCAAAGCCACAAGGGGAAAAA GGGACCAATGCATTCCTTCTCGTGAAGCATTTGCTCGCCAAAGCCAAAGCCAAAGCCAAAGCCACAAGGGGAAAAA GGGACCAATGCATTCCTTCTCGTGAAGCATTTGCTCGCCAAAGCCAAAGCCAAAGCCAAAGCCACAAGGGGAAAAA GGGACCAATGCATTCCTTCTCGTGAAGCATTTGCTCGCCAAAGCCAAAGCCAAAGCCAAAGCCACAAGGGGAAAAA GGGACCAATGCATTCCTTCTCGTGAAGCATTTGCTCGCCAAAGCCAAAGCCAAAGCCAAAGCCACAAGGGGAAAAA

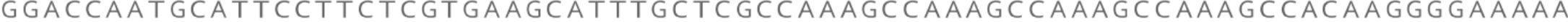
A
1,750
1,760
1,770
1.780
1,790
1,800

1,810

1,820

GAGA GA AA G GA G G CGTAGAACCGGAAAGGGCTCTGCATGTCTACCTTTCGCTGTGACCAAAATTACTCTTCCCT GAGAGAGAAGGAGGCGGTAGAACCGGAAAGGGCTCTGCATGTCTACCTTTCGCTGTGACCAAAATTACTCTTCC CT GAGAGAGAAGGAGGCGGTAGAACCGGAAAGGGCTCTGCATGTCTACCTTTCGCTGTGACCAAAATTACTCTTCCCT GAGAGAGAAGGAGGCGGTAGAACCGGAAAGGGCTCTGCATGTCTACCTTTCGCTGTGACCAAAATTACTCTTCCCT GAGAGAGAAGGAGGCGGTAGAACCGGAAAGGGCTCTGCATGTCTACCTTTCGCTGTGACCAAAATTACTCTTCCCT GAGAGAGAAGGAGGCGGTAGAACCGGAAAGGGCTCTGCATGTCTACCTTTCGCTGTGACCAAAATTACTCTTCC GAGAGAGAAGGAGGCGGTAGAACCGGAAAGGGCTCTGCATGTCTACCTTTCGCTGTGACCAAAATTACTCTTC C C GAGAGA GAAGGAGGCGGTAGAACCGGAAAGGGCTCTGCATGTCTACCTTTCGCTGTGACCAAAATTACTCTTC C C

$$
1,830
$$

1,860

1,870

1,880

1890

TCCGTGGCCCTGCTGCATGAGCATGGCCCTCCGCTTTTCAACCAGCAAGACAAGAATATGTCGCAATTGCTCCTAC TCG TCCGGGCCCTGCTGCATGAGCATGGCCCTCCGCTTTTCAACCAGCAAGACAAGAATAGTCGCAATTGCTCCTAC TCCTGCCCA TCA TCA TCCGTGGCCCTGCTGCATGAGCATGGCCCTCCGCTTTTCAACCAGCAAGACAAGAATATGTCGCAATTGCTCCTAC TCCGTGGCCCTGCTGCATGAGCATGGCCCTCCGCTTTTCAACCAGCAAGACAAGAATATGTCGCAATTGCTCCTAC 1,910 1,920 1,930 1,940 1,950 1,960 1,970 TAGTGTTAAACCGATGGATCAATCCTCCAAAATGCATCCCCCAACCAGACCCAACTTGCGCATAAACATCAATGTG

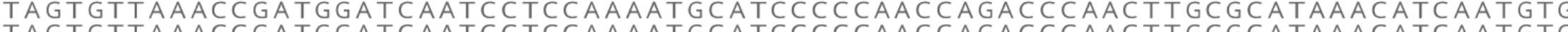
TAGT TAGTGTTAAACCGATGGATCAATCCTCCAAAATGCATCCCCCAACCAGACCCAACTTGCGCATAAACATCAATGTG TAGTGTTAAACCGATGGATCAATCCTCCAAAATGCATCCCCCAACCAGACCCAACTTGCGCATAAACATCAATGTG TAGTGTTAAACCGATGGATCAATCCTCCAAAATGCATCCCCCAACCAGACCCAACTTGCGCATAAACATCAATGTG TAGTGTTAAACCGATGGATCAATCCTCCAAAATGCATCCCCCAACCAGACCCAACTTGCGCATAAACATCAATGTG TAGTGTTAAACCGATGGATCAATCCTCCAAAATGCATCCCCCAACCAGACCCAACTTGCGCATAAACATCAATGTG 
dentity

1. Sloop

2. Morex

3. TR306

4. TR251

5. Logan

6. Harringto

7. CDC Bold

8. Beka

Identity

1. Sloop

2. Morex

4. TR251

5. Lan

6. Harringto

7. CDC Bold

8. Beka

Identity

1. Sloop

2. Morex

3. TR306

4. TR251

5. Logan

6. Harrington

7. CDC Bold

8. Beka

Identity

1. Sloop

2. Morex

3. TR306

5. Logan

6. Harrington

7. CDC Bolc

TAAAATTCCATGGACAGAAGAGAGCGCCGCATCGCATTTGCGGTGGGTGCAAGGCCACAGCGGAGCAAAGAG

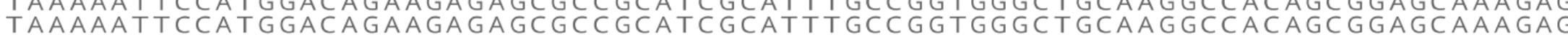
CTAAAATTCCATGGACAGAAGAGAGCGCCGCATCGCATTTGCCGGTGGGCTGCAAGGCCACAGCGGAGCAAAGAG CAAAATTCCATGGACAGAAGAGAGCGCCGCATCGCATTTGCCGGTGGGCTGCAAGGCCACAGCGGAGCAAAGAG


CTAAAAATTCCATGGACA GAAGA GAGCGCCGCATCGCATTTGCCGGTGGGCTGCAAGGCCACAGCGGAGCAAAGAG CTAAAAATTCCATGGACAGAAGAGAGCGCCGCATCGCATTTGCCGGTGGGCTGCAAGGCCACAGCGGAGCAAAGAG CTAAAAATTCCATGGACA GAAGA GA G G C C GCATCGCATTTGCCGGTGGGCTGCAAGGCCACA C G GAGCAAAGA 2,060 2,070 2,080 2,090 2,100 2,110 2,120

GCTTGTACGGTACTCAAACCAGCTCACTGATGCATGGCGATGCTACAGGGACTCACGGGCCACGCTGGCTGGGAGA GCTTGTACGGTACTCAAACCAGCTCACTGATGCATGGCGATGCTACAGGGACTCACGGGCCACGCTGGCTGGGAGA GCTTGTACGGTACTCAAACCAGCTCACTGATGCATGGCGATGCTACAGGGACTCACGGGCCACGCTGGCTGGGAGA

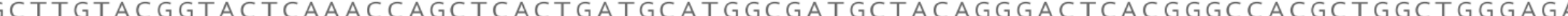

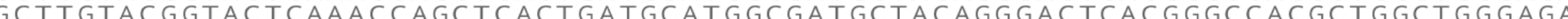
GCTTG G 2130 2,140 2.150 2160 2,170 2180 2,190 2200

GAGCAGCAGAAAAGCGCAAGAAAAGAATCTTTCTCTCAATCAAGGGCAACAACCAAGCACAAGCTCACAAACCCC 作 GAGCAGCAGAAAAGGCGAAGAAAAGAATCTTTCTCTCAATCAAGGGCAACAACCAAGCACAAGCTCACAAACCC
GAGCAGCAGAAAAGGCGCAAGAAAAGAATCTTTCTCTCAATCAAGGGCAACAACCAAGCACAAGCTCACAAACCCC

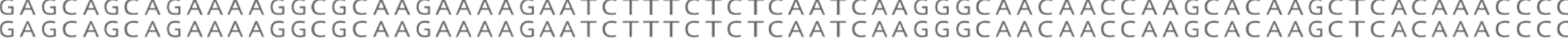
GAGCAGCAGAAAAGGCGCAAGAAAAGAATCTTTCTCTCAATCAAGGGCAACAACCAAGCACAAGCTCACAAACCCC GAGCAGCAGAAAAGGCGCAAGAAAAGAATCTTTCTCTCAATCAAGGGCAACAACCAAGCACAAGCTCACAAACCCC GAGCAGCAGAAAAGGCGCAAGAAAAGAATCTTTCTCTCAATCAAGGGCAACAACCAAGCACAAGCTCACAAACCC

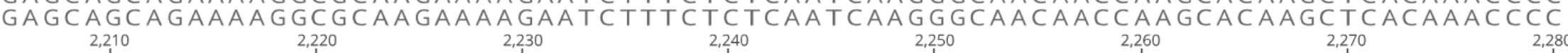
2,210

C CACCAAGTTAAGAAAAAGAAAAAAAAATACAGGCACTTCGCATAACAAACAAACTAATCATCATCACCACTCATC C CACCAAGTTAAGAAAAAGAAAAAAAAATACAGGCACTTCGCATAACAAACAAACTAATCATCATCACCACTCATC C CAC CAAGTTAAGAAAAAGAAAAAAAAATACAGGCACTTCGCATAACAAACAAACTAATCATCATCACCACTCATC C CACCAAGTTAAGAAAAAGAAAAAAAAATACAGGCACTTCGCATAACAAACAAACTAATCATCATCACCACTCATC $C \subset A C C A A G T T A A G A A A A A G A A A A A A A A A T A C A G G C A C T T C G C A T A A C A A A C A A A C T A A T C A T C A T C A C C A C T C A T C$ C CACCAAGTTAAGAAAAGAAAAAAAATACAGGCACTTCGCATAACAAACAAACTAATCATCATCACCACTCATC C CACCAAGTTAAGAAAAAGAAAAAAAAATACAGGCACTTCGCATAACAAACAAACTAATCATCATCACCACTCATC CCACCAAGTTAAGAAAAAGAAAAAAAAATACAGGCACTTCGCATAACAAACAAACTAATCATCATCACCACTCATC 
Identity

1. Sloop

2. Morex

3. TR306

4. TR251

5. Logan

6. Harrington

7. CDC Bold

8. Beka

Identity

1. Sloop

2. Morex

3. TR306

4. TR251

5. Logan

6. Harrington

7. CDC Bold

8. Beka

Identity

1. Sloop

2. Morex

3. TR306

4. TR251

5. Logan

6. Harrington

7. CDC Bold

8. Beka

Identity

1. Sloop

2. Morex

3. TR306

4. TR251

5. Logan

6. Harrington

8. Beka

GCCCCCGGCACCTTTATTCTACGCCCAGCTCCTCTCTCATTACACTTCTAACATATATAACAATCAATCGGTGGAG GCCCCCGGCACCTTTATTCTACGCCCAGCTCCTCTCTCATTACACTTCTAACATATATAACAATCAATCGGTGGAG GCCCCCGGCACCTTTATTCTACGCCCAGCTCCTCTCTCATTACACTTCTAACATATATAACAATCAATCGGTGGAG GCCCCCGGCACCTTTATTCTACGCCCAGCTCCTCTCTCATTACACTTCTAACATATATAACAATCAATCGGTGGAC GCCCCCGGCACCTTTATTCTACGCCCAGCTCCTCTCTCATTACACTTCTAACATATATAACAATCAATCGGTGGAG GCCCCCGGCACCTTTATTCTACGCCCAGCTCCTCTCTCATTACACTTCTAACATATATAACAATCAATCGGTGGAG GCCCCCGGCACCTTTATTCTACGCCCAGCTCCTCTCTCATTACACTTCTAACATATATAACAATCAATCGGTGGAG GCCCCCGGCACCTTTATTCTACGCCCAGCTCCTCTCTCATTACACTTCTAACATATATAACAATCAATCGGTGGAC 2,360 2,370 2,380 2,390 2,400 2,410 2,420 2,430

GA G GA GG GACCAATTAATTAATGGATTAGTAATAACAATTCTTATGCGAGCAAAAATATACGTACTCCTGTACTAA

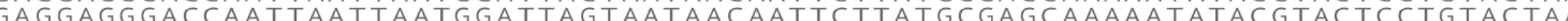
AAGGGGACCAATTAATTAATGGATTAGTAATAACAATTCTTATGCGAGCAAAAATATACGTACTCCTGTACTAA




GAGAGGGACCAATTAATTAATGGATTAGTAATAACAATTCTTATG AAGA

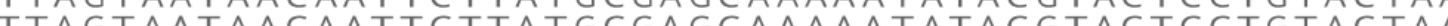
2,440 2,450 2,460 2,470 2,480 2,490 2,500

AAGAATCGGAAATGCAAGCTAATCAGA GCTACTGCGTACGTACTAGTTTTCTTGCGCGGGGAGGGCATCACAAAT TAAGAATCGGAAATGCAAGCTAATCAGAGCTACTGCGTACGTACTAGTTTTCTTGCGCGGGGAGGGCATCACAAAT TAAGAATCGGAAATGCAAGCTAATCAGAGCTACTGCGTACGTACTAGTTTTCTTGCGCGGGGAGGGCATCACAAAT AAAAATCGGAAATGCAAGCTAATCAGAGCTACTGCGTACGTACTAGTTTTCTTGCGCGGGGAGGGCATCACAAAT


TAAGAATCGGAAATGCAAGCTAATCAGAGCTACTGCGTACGTACTAGTTTTCTTGCGCGGGGAGGGCATCACAAAT TAAGAATCGGAAATGCAAGCTAATCAGAGCTACTGCGTACGTACTAGTTTTCTTGCGCGGGGAGGGCATCACAAAT TAAGAATCGGAAATGCAAGCTAATCAGAGCTACTGCGTACGTACTAGTTTTCTTGCGCGGGGAGGGCATCACAAAT 2,510 2,530 2,540 2,550 2,560 2,580

CACATGGGACACGCAGGGGCAGGAATATGAGTGGAAGCAACCTGGTAGGTAGGTGAGGTGCGGTGCGGTGCGGGCA CACATGGGACACGCAGGGGCAGGAATATGAGTGGAAGCAACCTGGTAGGTAGGTGAGGTGCGGTGCGGTGCGGGCA CACATGGGACACGCAGGGGCAGGAATATGAGTGGAAGCAACᄃTGGTAGGTAGGTGAGGTGCGGTGCGGTGCGGGCA $\subset A \subset A T G G G A \subset A \subset G \subset A G G G G \subset A G G A A T A T G A G T G G A A G \subset A A \subset \subset T G G T A G G T A G G T G A G G T G C G G T G \subset G G T G \subset G G G \subset A$ CACATGGGACACGCAGGGGCAGGAATATGAGTGGAAGCAACᄃTGGTAGGTAGGTGAGGTGCGGTGCGGTGCGGGCA CACATGGGACACGCAGGGGCAGGAATATGAGTGGAGCAACC CACATGGGACACGCAGGGGCAGGAATATGAGGG $\subset A \subset A T G G G A \subset A \subset G \subset A G G G G \subset A G G A A T A T G A G T G G A A G \subset A A \subset \subset T G G T A G G T A G G T G A G T G C G G T C G G T G \subset G G G A$ 
dentity

1. Sloop

4. TR251

5. Logan

6. Harrington

7. CDC Bold

8. Beka

Identity

1. Sloop

2. Morex

3. TR306

5. 2251

5. Logan

7. CDC Bold

8. Beka

Identity

1. Sloop

2. Morex

3. TR306

4. TR251

5. Logan

6. Harrington

7. CDC Bold

8. Beka

Identity

1. Sloop

2. Morex

3. TR306

5. TR251

5. Logan

7. CDC Bold

7. CDC B
8. Beka

TATGATAAGCATATGGGCTGGGCCGAGCATACACGCGTACATGCATTGCATTTGCATACACCGAAGGAAGTGCTTG TATGATAAGCATATGGGCTGGGCCGAGCATACACGCGTACATGCATTGCATTTGCATACACCGAAGGAAGTGCTTG TATGATAAGCATATGGGCTGGGCCGAGCATACACGCGTACATGCATTGCATTTGCATACACCGAAGGAAGTGCTTG TATGATAAGCATATGGGCTGGGCCGAGCATACACGCGTACATGCATTGCATTTGCATACACCGAAGGAAGTGCTTG TATGATAAGCATATGGGCTGGGCCGAGCATACACGCGTACATGCATTGCATTTGCATACACCGAAGGAAGTGCTTG TATGATAAGCATATGGGCTGGGCCGAGCATACACGCGTACATGCATTGCATTTGCATACACCGAAGGAAGTGCTTG


2,670 




